\title{
Scaffolding Stakeholder-centric Enterprise Model Articulation
}

\author{
Stefan Oppl ${ }^{1}$, Stijn Hoppenbrouwers ${ }^{1,2}$, \\ ${ }^{1}$ Radboud University, Department of Software Science, Toernooiveld 212 \\ 6525 EC Nijmegen, Netherlands \\ stefan.oppl@jku.at \\ ${ }^{2}$ HAN University of Applied Sciences, Model-Based IS Group, Ruitenberglaan 26 \\ 6802 CE Arnhem, Netherlands \\ stijn.hoppenbrouwers@han.nl
}

\begin{abstract}
Involving stakeholders in enterprise modeling, besides rendering valid models, also helps stakeholders articulate and align their views on their organization. This requires that stakeholders are able to understand and actively perform conceptual modeling for representing their views on enterprise structure and behavior. The specific skills required for this should not be taken for granted and need to be developed explicitly. Scaffolding is an educational concept that allows to embed learning support mechanisms in operative modeling processes. The present article introduces a framework that makes it possible to view scaffolding as an integral part of stakeholder-centric modeling activities. The framework is validated with respect to its descriptive and discriminatory power by an ex-post analysis of the design and application of an existing modeling method.
\end{abstract}

Keywords: scaffolding, stakeholder-centric modeling, articulation, alignment

\section{Introduction}

Enterprise modeling is a form of conceptual modeling that is concerned with representing organizational phenomena and their relationships among each other [1]. The developed conceptual models provide the foundation for describing present or required [2] properties of enterprises as socio-technical systems, to document their operational and structural properties [3] and aid the communication with social and technical stakeholders throughout the design process [4].

The topic of how to facilitate the development of skills in conceptual modeling in organizational research has been addressed as early as in the 1960s, when Morris [5] stated that ,if one grants that modeling is and, for greatest effectiveness, probably ought to be, an intuitive process for the experienced, then the interesting question becomes the pedagogical problem of how to develop this intuition." This question has also been picked up in enterprise modeling as the discipline continued to mature [6], and has moved away from being considered an „art“ that requires „intuition“ to a more scientifically grounded discipline [7].

The work of Stefan Oppl has been supported by the Austrian Science Fund (FWF): $\mathrm{J}-3882$ 
In recent years, literature examining conceptual model processes [8] [9] recognizes a trend towards a strong and active involvement of stakeholders, who are usually not formally trained in modeling [10]. Models are considered to act as boundary objects [11] that enable people to articulate and align their understanding of their work systems [12]. Research in this domain has focused on how to facilitate modeling activities under involvement of such „,novice modelers“ [8] for generating models appropriate for the respective aims of modeling [13]. In contrast, the question of how to support the development of skills to work with and on the basis of conceptual enterprise models for this group of people, who usually does not have the opportunity to dedicate effort and time to formal modeling education, has hardly been a subject of research. The potential added value of such skills for this group, however, has been recognized repeatedly over the last decades in terms of pursuing a deeper understanding of the domain and phenomenon being subject of modeling $[3,13,14]$.

The aim of the present work is to address this issue by making a step towards a framework for embedding scaffolding (offering help and guidance for as long as necessary; see 2.2) in stakeholder-oriented enterprise modeling to enable stakeholders to directly contribute to modeling. Our framework aims at enabling theory-informed method design. We thus methodologically follow Walls et al. [15], and identify metadesign requirements based on our kernel theories, derive a meta-design based on these requirements and give a first validation of our framework by ex-post analysis of a workshop design for stakeholder-oriented enterprise modeling. The contribution of the present work is that it augments current practices for enterprise model elicitation with an explicit skill development perspective that aims at enabling stakeholders to actively participate in modeling.

This paper is structured as follows: In the next section, we elaborate on background and related work to establish the foundation for our framework. The meta-requirements in terms of embedding scaffolding in modeling workshop designs are established in section 3 and are encoded in a framework that serves as the meta-design. Section 4 validates this framework on two levels: we first use it to conduct an ex-post analysis of an existing modeling method and second deploy it as a lens for analyzing concrete workshops from a learning support perspective. We close with a discussion of our findings and derive directions of future research.

\section{Background \& Related Work}

In this section we introduce the background theories and related work our research is based on. This establishes the research framework of the present work and allows to derive design guidelines for scaffolds in the following section.

\subsection{Enterprise Modeling as a Form of Stakeholder Articulation}

The creation of enterprise models under the involvement of stakeholders not only is a form of model elicitation [6], but - if performed collaboratively in a group of stakeholders - can be considered a form of Articulation Work [16]. Articulation Work 
refers to all activities concerned with setting up and maintaining cooperative work in organizations (ibid.). Articulation work in most cases happens implicitly and is triggered during the ongoing productive work activities whenever contingencies arise. When aligning views on organizational work with those of others and aligning them to form a coherent, normative model of enterprise structure and behavior [17], articulation work takes a more explicit form that allows to establish work practices or structures beyond the immediate context of the group of involved people [18]. Enterprise modeling as a form of creating external artifacts to represent organizational phenomena in conceptual models is a means to support such activities [19]. The potential added value of enterprise modeling activities in that respect, however, can only be realized if the stakeholders are able to understand and use the respective means of representation [20]. The availability of the necessary skills must not be taken for granted [21] and must be developed explicitly [22]. Based on existing empirical evidence [23], we hypothesize that support for the development of these skills can be embedded in the modeling process by means of scaffolding.

\subsection{Scaffolding}

Scaffolding is a concept introduced in the field of educational tutoring by Wood et al. [24]. It originally refers to having an experienced person help an unexperienced learner to acquire knowledge about a particular topic. Scaffolding is a metaphor adopted from construction industry and refers to a temporary means of support that is present until the entity supported by scaffolds (here: a subject participating in conceptual modeling) can accomplish a given task herself [25]. It is usually motivated by the aim of keeping subjects in their ,zone of proximal development“ (ZPD) during a learning process [26], i.e., putting them in a situation, which is challenging, yet attainable to them. In order for scaffolds to be acceptable for subjects and provide added value to them, they need to be appropriated to their current skill level [27].

Scaffolding can take different forms. Based on a meta-study of scaffolding research, Jumaat \& Tasir [28] distinguish conceptual scaffolds, procedural scaffolds, metacognitive scaffolds and strategic scaffolds. Conceptual scaffolds help learning to decide what to consider to be worth learning. In particular, they can help to prioritize fundamental concepts. Procedural scaffolds assist students in using available tools and methods and point them at potentially useful resources. Strategic scaffolds suggest alternative ways to tackle problems in learning. Finally, metacognitive scaffolds guide students in how to approach a learning problem and what to think about when elaborating on a problem. Orthogonally to these categories, Bulu \& Pedersen [29] identify differences in the sources of scaffolding. Scaffolds provided by teachers are considered the original form of scaffolding. Scaffolds provided in interactions among learning peers refer to the phenomenon that scaffolding can arise from the collective knowledge of a learning group. Scaffolds can also be provided as textual or graphical representations, similar to a manual. Technology-driven scaffolding uses (information) technology to provide scaffolds. This includes interactive systems that try to intervene appropriately in the learning process based on observing learners' behaviors or static intervention rules. 
Independently of which form of scaffolding is pursued, it is always characterized via the presence of three principles that have been identified by van de Pol et al. [25]: The first common principle is contingency, which is often referred to as responsiveness or calibrated support. Scaffolds need to be adapted dynamically to the learners' current level of performance. The second principle is fading, which refers to the gradual withdrawal of the scaffolding. As learners develop their skills, support becomes less necessary and is decreased over time. This is closely connected to the third principle transfer of responsibility. Via fading, responsibility for the performance of a task is gradually transferred to the learner. The responsibility for learning is transferred when a student takes increasing control about the learning process. The implementation of these principles is based on diagnosis of a learners' need for support, which is usually done by a teacher [23], but also can be implemented in interactive systems [30].

On an operative level, scaffolding is implemented via different means. Van de Pol et al. [25] list a (non-exhaustive) set of measures such as giving feedback, providing hints, instructing, explaining, modeling (i.e. demonstrating the skill to be acquired) and questioning. They differ in their depth of intervention and the reduction of freedom in students' learning processes. How to appropriately select and implement scaffolding as interventions in the learning process is disputed (ibid.). The described categories and means thus should be considered a framework for observing and designing learning settings, rather than attribute them any normative value.

\subsection{Related Work}

In the context of conceptual modeling, several authors have examined how scaffolds can be used to facilitate the modeling process.

Fretz et al. [31] propose a software tool that provides conceptual and procedural scaffolds to subjects confronted with a conceptual modeling tasks in the context of science learning. In addition, they examine additional strategic and procedural scaffolds provided by teachers or peers. They use prompting to trigger reflection of modeled structures and allow to validate the model by interactive simulations. As such, the tool uses scaffolds to implement a setting that facilitates argumentative discourse via procedural guidance. The authors do not refer to principles like fading or transfer of responsibilities, but rather focus on examining the effect of the different types of scaffolds in the argumentative design. They found that - although the designed scaffolds achieved their intended effects - the role of teachers and peers in association with designed scaffolds appears to be vital for successful deployment.

Sandkuhl et al. [32] and Carstensen et al. [33] use the term scaffolding in the context of enterprise modeling, but refer to it in an architectural sense. They aim at creating "shared knowledge and understanding" among subjects in a collaborative modeling session before the actual problem-solving process starts. While this is fundamentally different from the understanding of scaffolding put forward above, the means they propose still can inform scaffold design for conceptual modeling. In particular, they use metacognitive scaffolds encoded in graphical modeling templates that indicate which perspectives should be considered relevant for a particular enterprise modeling problem. 
Oppl [34] has reviewed the state of the art of conceptual modeling workshop facilitation techniques with a focus on how scaffolding concepts are used to support modeling processes. The results indicate that in particular metacognitive and procedural scaffolds are regularly used for facilitation, usually in an implicit way, without referring to scaffolding as such. They also show that hardly any existing approaches give an explicit account on the fundamental scaffolding principles: contingency, fading, and transfer of responsibility. Related work thus currently does not enable to systematically embed scaffolding measures in modeling workshop design. We thus derive a framework to address this issue from research performed on scaffolding for supporting collaborative articulation in general in the next section.

\section{Scaffolds for Stakeholder-centric Enterprise Modeling}

Our review of related work has shown that, while scaffolding has already implicitly and, to some extent, explicitly been deployed in the field of conceptual modeling, a structured approach to describe and design scaffolding in modeling activities is not available. As argued for in the introduction, augmenting the design of stakeholdercentric modeling activities with a scaffolding perspective could help improve the understanding and creation of enterprise models in the target group. In the following, we therefore review scaffolding approaches proposed in other disciplines, which require similar skills as stakeholder-centric conceptual modeling, in particular with respect to articulation of abstract concepts describing real-world phenomena [21] and the support of developing a common understanding about these phenomena [35]. Based on these approaches, we develop a framework for scaffolding in enterprise modeling.

\subsection{Scaffolding the articulation of models}

The process of articulating abstract concepts, being a main activity in conceptual modeling, has been widely examined regarding potential scaffolding support in the field of mathematical and science education.

Ozmantar \& Roper [36] consider teacher interventions as the major means of scaffolding (in the context of mathematical abstraction problems). Their study focusses on examining the activities of the person providing scaffolds. They identify three major facets that they could observe. First, they observed that scaffolding strategies were chosen in an ad-hoc manner based on continuous monitoring and analyzing the subjects' performance. Which scaffold is appropriate in which situation needs (and they pose - cannot) be determined ex-ante. However, continuous monitoring allows to analyze, whether a provided scaffold achieves the intended effect and eventually adapting ones scaffolding strategy. Second, they identify a major means of scaffolding to provide meta-cognitive scaffolds by organizing the main goal of the learning activity into hierarchical sub-goals. Third, they could observe fading and transfer of responsibility to take place when models went beyond their initial construction and had begun to stabilize via consolidation activities. 
Land \& Zembal-Saul [37] examine how reflection and articulation processes on scientific explanations can be scaffolded. This focus is conceptually close to what other authors refer to as conceptual modeling. They examine means that to scaffold reflection and articulation on a longer-term time scale and focus on means of scaffolding via peers. Their scaffolds are deployed via a software platform, and are mainly metacognitive, based on task-specific prompting. They could show that their design was useful to learners and led to sophisticated explanations, indicating the construction of elaborate abstractions. They, however, found that the utility of "static" scaffolds as provided by their platform was dependent on the background knowledge of the learners. They thus suggest to combine their approach with human instruction that provides more explicit scaffolding especially for novices.

Stender \& Kaiser [23] discuss the value of scaffolding the process of developing mathematical models of real-world-problems and validate their appropriateness for problem-solving. Rather than describing concrete scaffolds, they focus on diagnosis of student needs and fading support measures to facilitate independent problem-solving by students. Based on existing research on adaptive teacher interventions, they identify the invasiveness of different types of scaffolding in terms of restricting student's freedom of choice on action. Based on their empirical results, they suggest scaffolding interventions in the model articulation process to facilitate problem solving. Their results indicate, among others, the usefulness of decomposition of the modeling task, availability of model simulation tools and referring to existing knowledge via metacognitive scaffolds.

\subsection{Scaffolding argumentative collaboration}

Several authors have also examined how to scaffold collaborative articulation, in particular with focus on peer-facilitation of argumentative processes.

Abdu et al. [38] examine how the process of peer-facilitation can be scaffolded with whole-group interventions in classroom settings. Their focus consequently is on argumentative design that should prevent unguided model creation. They propose to provide strategical scaffolds by means demonstrating solution paths upfront, before peer interaction starts. Furthermore, they establish explicit prompting practices for peer collaboration to establish collective responsibility for the learning process.

Chin \& Osborne [39] show how discursive interaction (i.e. argumentation) can be scaffolded in science education. They propose to provide question prompts for enabling peers to ask questions that allow to explore a problem or proposed solution more in depth [40]. They propose to provide conceptual scaffolds in the form of additional resources on the topic of interaction and procedural scaffolds in the form of guiding structures (such as writing stems or diagram templates). They furthermore propose to work with heterogeneous groups, where participants have different views, to facilitate confrontative argumentation [35].

Xun \& Land [41] focus on the development of domain-specific question prompts to scaffold problem-solving in peer interaction settings. They establish guidelines for designing such scaffolds that are based on a combination of generic discursive prompting [40] and domain-specific prompts that they claim should be developed in 
cooperation with domain experts. They also suggest to structure discourse via explicit assignment of roles to learners they should take in their interaction.

Vogel et al. [42] present a meta-study on how collaboration scripts can be used for scaffolding in IT-supported learning environments. Collaborations scripts [43] are strategic scaffolds that specify a sequence of learning activities to be completed to achieve the aim of a particular task. They found that collaboration scripts have positive effects on the acquisition of domain-specific knowledge in relation to the task and collaboration skills in general. They claim that repeated participation and practice in activities scaffolded by collaboration scripts leads to an internalization of the required performative knowledge and gradually allows to withdraw the script guidance while still maintaining the developed problem-solving strategies not only in terms of collaboration, but also regarding domain-specific skills.

\subsection{A Framework for Scaffolding Model Articulation}

Based on the empirical results presented in $[23,37,42]$ and informed by the methodological considerations outlined in $[36,38,39,41]$, we hypothesize that scaffolds can be developed for the purpose of skill development in stakeholder-centric modeling settings. The modeling process in this context is considered a process of articulation. It is indivisibly embedded in its social context that requires to view conceptual modeling as a process of co-construction ultimately leading to a shared understanding about the topic of modeling among the participating subjects. Consequently, a conceptual model always only can represent the agreed upon abstractions of the perceived real world phenomena considered relevant by the participants. Its value is further determined by the chosen representational system, that needs to be selected based on the intended purpose, i.e. goal of modeling.

Informed by the prior research discussed above, modeling approaches from a scaffolding perspective need to address the following meta-requirements [15]: (A1) provide scaffolds for the level of model representation (i.e. encoding abstractions in an external representation) [23, 37], (A2) provide scaffolds for the level of model articulation (i.e. developing an understanding about the real world phenomenon that is the topic of modeling) [23, 41], and (A3) provide scaffolds for the level of collaborative model alignment (i.e. the process of mutually supporting the development of a shared understanding about the topic of modeling and the modeling process itself) [38, 39, 42]. The identified meta-requirements A1-A3 link issues discussed in the field of stakeholder-centric model articulation[18-20] with potential support measures provided by scaffolding practices.

Furthermore, in order to account for the aim of supporting modeling skill development $[21,22]$ and allow for contingency, fading and transfer of responsibility, (A4) scaffolds need to be provided with different degrees of invasiveness to allow to adapt modeling support to the current needs of the modelers [36] [42].

Based on these requirements, we draw from the results of our literature review in the following and propose a meta-design [15] in the form of a scaffolding framework which should support the process of enterprise modeling on all three levels identified above. The framework is visualized in Figure 1. 
The fundamental constituents of the framework are visualized on the top, bottom and left margin of Figure 1. Its starting point can be found on the left, where our conceptualization of enterprise modeling being activities of a group of individuals (stakeholders) to create a common conceptual model is shown. As identified above, this requires to perform model representation, model articulation and model alignment activities, and is usually supported by a facilitator.

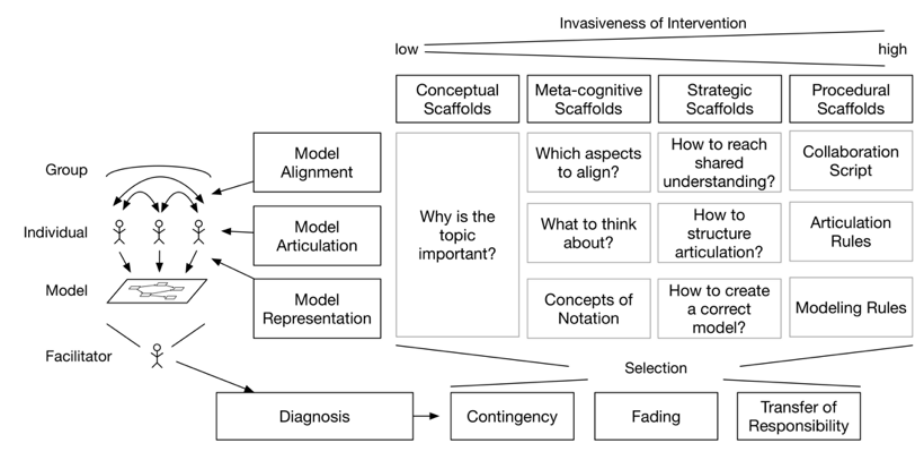

Fig. 1. Dimensions of Scaffolding during Enterprise Modeling

The top margin of Figure 1 structures different types of scaffolds [25] according to their invasiveness of intervention [23] (cf. A4). Depending on the diagnosis of current needs of the group of stakeholders engaged in modeling, the facilitator is deploying different types of scaffolds following the principles of scaffolding visualized at the bottom margin of Figure 1. The more responsibility is transferred to the modelers, the more support measures are faded out, and scaffolds are deployed (if any) that are less invasive. In case of contingency, the facilitator is free to temporarily fade in stronger support to keep the modelers oriented towards the aim of modeling.

The center area of Figure 1 shows the aspects of modeling that should be addressed by different types of scaffolds for model representation (cf. A1), articulation (cf. A2) and alignment (cf. A3). In general, conceptual scaffolds independently of the addressed level motivate the topic of modeling, show its relevance and allow to validate the model with respect to their appropriateness in real-world use. Metacognitive scaffolds support to understand the structure of the modeling task and indicate how conceptualize the real-world phenomenon. On the level of model alignment, metacognitive scaffolds indicate which aspects of a model are subject to alignment (i.e. interfaces between model parts, in contrast to those aspects that remain in the responsibility of individual modelers). Strategic and procedural scaffolds aim at supporting the modeling process itself, either by showing potential behavioral strategies in the first case or providing stricter guidance in the second case. On the level of model representation, such scaffolds focus on syntactic aspects of modeling, whereas articulation and alignment focus on semantic aspects.

Scaffolds of either form and on either level can be delivered via different channels. They can be provided by procedural guidance or by artifacts (static or interactive media) designed to mediate the modeling process. Procedural guidance can be provided 
by a human facilitator or an IT-based system, if the latter is capable of monitoring the modeling progress and analyze the challenges participants currently face on their individual skill level. Procedural guidance by humans can be provided by expert facilitators or peers, if the latter are provided with further scaffolds, that guide the facilitation process itself. Designed artifacts can be provided for domain-specific support and for collaboration support, whereas in both cases their value is determined by an anticipated fit between the skill level of the addressed subjects and the support provided by the artifact. As this fit usually cannot be taken for granted, pre-designed scaffolds are usually combined with a form of procedural guidance.

Figure 2 gives examples for these different types of scaffolds, distinguishing between different sources of scaffolding as described in section 2.2. The examples are taken from related work (references provided for each example in the figure).

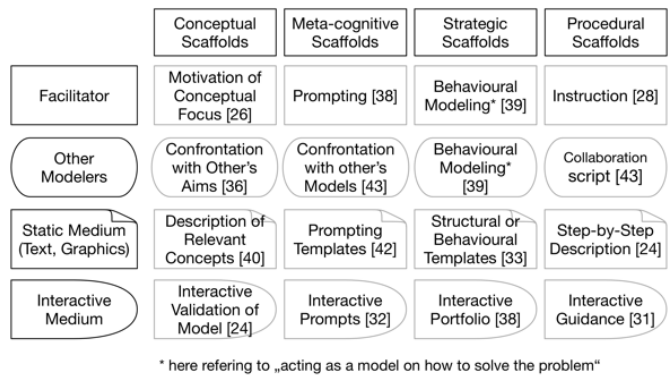

Fig. 2. Examples of different forms of scaffolds for enterprise modeling as described in related work

The examples should be considered a non-tentative overview about how the different forms of scaffolding can be provided via different delivery channels. They are deliberately not assigned to the different levels of support indicated in Figure 1 (model representation, model articulation, model alignment), as existing literature does not distinguish between these levels. For validation, we combine the delivery channels with the forms of scaffolding and levels of support for an actual method (cf. Figure 3).

\section{Validation}

We validate the framework developed above by using it for analysis of modeling method designs. This analysis examines the framework's descriptive and discriminatory value and its usefulness for assessing observed modeling processes with a scaffolding lens. We hypothesize that the framework enables a structured review of support measures embedded in modeling method designs and provides an analytical lens for observed modeling processes that allows to analyze potential reasons for the success (or lack thereof) of stakeholder-centric modeling workshops.

Based on the meta-requirements A1-A4 formulated in section 3.3, we formulate testable hypotheses (designated with Hx.y in the following) for assessing the appropriateness of the proposed framework [15]. In ex-post analysis of modeling 
method designs, we hypothesize that (H1.1) the support measures embedded in the method are classifiable with respect to whether they target model alignment, model articulation, or model representation. Furthermore, (H1.2) the support measures are classifiable with respect to the form of scaffolding and the proposed delivery channel (facilitator, peer, static media, interactive media). In analysis of observed modeling processes, we hypothesize that $(\mathrm{H} 2.1)$ the observed support measures are classifiable with respect to whether they target model alignment, model articulation, or model representation. Furthermore, (H2.2) they are classifiable with respect to form and delivery channel of scaffolding. Finally, (H2.3) the observed behavior of the facilitator is classifiable with respect to properties of scaffolding implemented during modeling (contingency, fading, transfer of responsibility).

If any of the hypotheses could not be validated in the study, i.e., any method design property (for H1.x) or observed behavior (for H2.x) cannot be classified in the framework, this would indicate a conceptual deficiency in the framework that would need to be addressed in future refinements of the framework. If the hypotheses could be confirmed, this would indicate appropriate discriminatory power of the framework. If the results of workshop analysis (H2.x) furthermore allowed the linking to the deployed scaffolding measures of outcomes of the model representation, articulation and alignment (according to the respective aims of modeling), this would indicate appropriate descriptive power of the framework.

\subsection{Sample Instantiation in CoMPArE/WP}

H1.1 and H1.2 have been validated by ex-post analysis of the CoMPArE/WP method [44], which explicitly aims at supporting articulation and alignment of stakeholders' views on their contributions to enterprise processes and the collaboration necessary to implement them. The method follows a multi-step modeling approach, in which participants first collaboratively create a concept map to agree on the notions used to refer to the relevant aspects of their work, then individually model their views on their own contributions and interfaces with others, and finally consolidate these models in a discursive way to create an agreed-upon representation of the overall work process. If modeling rules are adhered to, the resulting models are technically interpretable by a workflow engine and in this way can be validated through simulated execution [44].

Although not designed with a scaffolding background, CoMPArE/WP offers support measures to enable modeling by stakeholders, who do not have any prior knowledge in conceptual modeling. These support measures are briefly described in the following and then classified using the proposed framework (cf. Figure 3). The global multi-step modeling procedure is introduced by a facilitator, who is expected to be trained in the implementation of the method. The participants are also provided with a one-page written/graphical summary of the global procedure. The used modeling notations are pre-specified and are provided via cardboard model elements that follow a coloring scheme encoding the semantics the used modeling language. The same coloring scheme is used in poster-sized printed templates that indicate the expected model layout that needs to be adhered to in order for the results to be unambiguously interpretable via technical means. Printed model examples are provided for reference in case of 
uncertainty on how to use the model elements or their semantics. The participants have access to written descriptions of the modeling rules for each step. During workshop implementation, the facilitator provides role-specific prompts to aid model development. If necessary, the facilitator demonstrates model development using an example. During consolidation, the participants are expected to contribute their individual models and support the identification of model parts that indicate divergent views on how to collaborate. The identification process is supported via the contributed models of all participants that should contain semantically identical model elements in case of agreement on how to collaborate. The resulting model is interactively validated via simulated enactment, during which participants can identify inadequacies of the developed model.

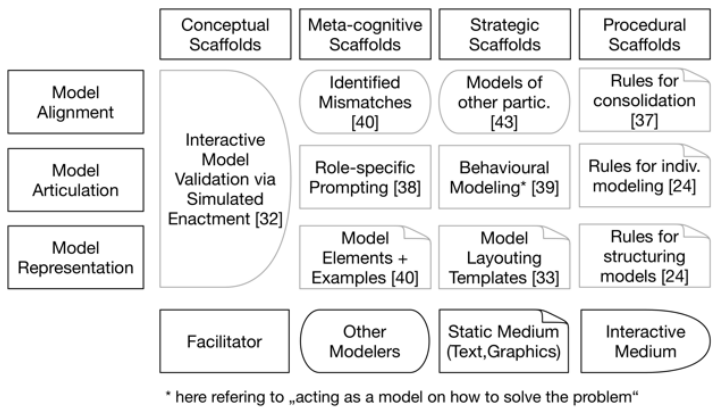

Fig. 3. Scaffolds deployed in CoMPArE/WP (references indicate the foundation for design)

Figure 3 shows a classification of the available support measures using the proposed scaffolding framework. With respect to the hypotheses formulated above, H1.1 can be confirmed with two exceptions: validation via simulated enactment cannot be classified to any particular level of support, as it potentially contributes to all three levels (showing the relevance of syntactic model correctness for representation, the impact of individual contributions to the model on articulation level, and the need for a commonly agreed model on alignment level). As this particular measure acts as a conceptual scaffold in the present method, classification in one specific level, however, is not mandatory in the framework. The global multi-step modeling procedure cannot be classified in the framework due to its more generic, level-independent scope. This indicates a shortcoming of the current framework.

H1.2 is not violated for the methodology, as each support measure could be classified with respect to its form of scaffolding and delivery channel. The global multi-step modeling procedure is not contained in Figure 3 due to the problems described for H1.1, but would pose a procedural scaffold delivered by the facilitator.

Using the framework to analyze CoMPArE/WP allows to identify the following potential shortcomings of the method: (1) the scaffolds for model alignment are largely based on peer delivery. However, no meta-scaffolds on how to provide scaffolds to peers are available and thus rely on prior existing knowledge on modeling, and (2) a conceptual scaffold is only available after initial modeling activities, as virtual enactment requires an initial model version. 


\subsection{Sample Workshops}

$\mathrm{H} 2.1, \mathrm{H} 2.2$, and H2.3 have been validated by ex-post analysis of three workshops that were implemented following the CoMPArE/WP method. The workshops were selected to differ in the amount of scaffolds deployed and the behavior of the facilitator to comprehensively assess the framework's elements. Any scaffold that had been deployed was implemented in the way envisaged by the method. Consequently, as the method has already satisfied H1.1 and H1.2, the observable scaffolds in the method's implementation also satisfy H2.1 and H2.2. Differences here could only be anticipated, if the workshop implementation deviated from its design.

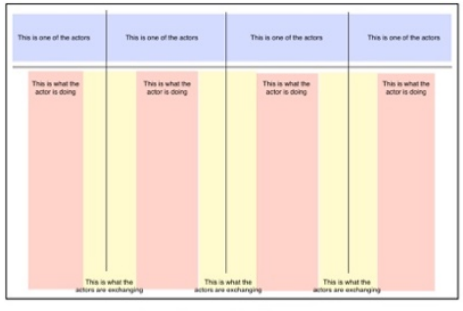

Model Layouting Template

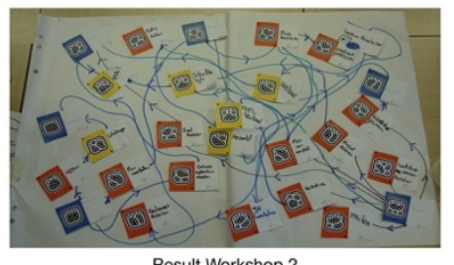

Result Workshop 2

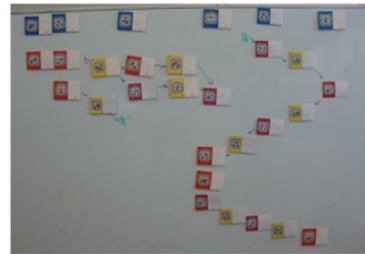

Result Workshop 1

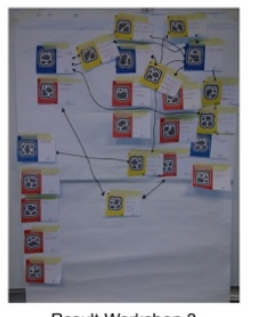

Result Workshop 3

Figure 4: top left: model layout template, top right $\&$ bottom: modeling results of workshops

$\mathrm{H} 2.3$ is discussed in the following by example of the observable effects caused by how the facilitators guided model representation and alignment. Figure 4 shows a model layout template which is used as a strategic scaffold for model representation during consolidation. The three photos in Figure 4 illustrate the different results of consolidation. On representation level, the aim is to resemble the layout indicated in the template (blue elements on top, red elements aligned below in lanes, yellow elements placed between lanes). On alignment level, participants themselves should discover problems in the depicted process (e.g., non-matching communication expectations) and resolve them.

The facilitator in workshop 1 deployed model representation scaffolds on a strategic and procedural level. Scaffolds for model articulation and alignment were not used. Fading, transfer of responsibility or contingency could not be observed. This resulted in a syntactically correct model, but led to little involvement of the stakeholders in modeling and articulation and no observable alignment activities. The facilitator in workshop 2 introduced the global multi-step modeling procedure as a high-level procedural scaffold and provided the participants with meta-cognitive scaffolds on representation, articulation and alignment. Contingency could not be observed, 
although participants showed signs of being overwhelmed with the task. He rather shifted full responsibility to them after an initial deployment of the metacognitive scaffolds. While high involvement of all participants in articulation could be observed, participation was declining during alignment, and led to a syntactically incorrect modeling result with semantic deviations from the proposed modeling language. The facilitator in workshop 3 actively implemented contingency and fading. She started with strategic scaffolds for model representation and articulation, briefly provided procedural scaffolds at the start of the consolidation step and provided metacognitive scaffolds in case of contingency. The observed modeling process continuously showed high involvement in articulation and alignment, with deviations from the proposed modeling notation in the final modeling result.

$\mathrm{H} 2.3$ is backed by these results, as classification of the observed behavior of the facilitators was possible with respect to properties of scaffolding implemented during modeling (contingency, fading, transfer of responsibility). Furthermore, their behavior could be described using the addressed levels of support, forms of scaffolding and their delivery channels. This indicates adequacy of the hypotheses $\mathrm{H} 2.1$ and H2.2.

\section{Discussion and Conclusion}

The results of evaluation in section 4 show that the hypotheses formulated based on the identified meta-requirements could be confirmed. Shortcomings in the current version of the framework could be identified with respect to scaffolds that span different levels of support or support the modeling process on a generic level. The findings with respect to the hypotheses H1.x provide evidence that the framework could potentially guide modeling method design from a scaffolding perspective. The findings on hypotheses H2.x indicate that further examination of the framework's potential for training facilitators to provide situation-specific support to stakeholders [13] appears to be worthwhile. The present article has thus introduced a framework on anchoring scaffolding in stakeholder-centric enterprise modeling. It indicates relevant dimensions of design when augmenting modeling activities with scaffolding.

The current state of development of the framework suffers from several limitations that need to be addressed in future research. First, the framework currently lacks instantiation guidelines [15] to apply it in modeling method design. This will be addressed in the next revision of the framework. Second, the validation study currently provides only limited internal validity, as only one method has been examined in this article. Our current research, however, indicates that the framework can be also applied to other methods and exhibits similar shortcomings there.

These limitations will be addressed in our future research by establishing a more comprehensive empirical basis for the next iteration of our framework. Our next steps will go beyond analytical deployment of the framework and will examine whether it can be used to guide method design in practice. 


\section{References}

1. Sandkuhl, K., Stirna, J., Persson, A., Wißotzki, M.: Enterprise Modeling.

2. Insfrán, E., Pastor, Ó., Wieringa, R.: Requirements Engineering-Based Conceptual Modelling. Requirements Eng. 7, 61-72 (2002).

3. Davies, I., Green, P., Rosemann, M., Indulska, M., Gallo, S.: How do practitioners use conceptual modeling in practice? Data $\backslash \&$ Knowledge Engineering. 58, 358-380 (2006).

4. Heemskerk, M., Wilson, K., Pavao-Zuckerman, M.: Conceptual models as tools for communication across disciplines. Conservation Ecology. 7, 8 (2003).

5. Morris, W.T.: On the art of modeling. Management Science. 13, B-707-B-717 (1967).

6. Sandkuhl, K., Stirna, J., Persson, A., Wißotzki, M.: Elicitation Approaches in Enterprise Modeling. In: Enterprise Modeling. pp. 39-51. Springer (2014).

7. Willemain, T.R.: Model formulation: What experts think about and when. Operations Research. 43, 916-932 (1995).

8. Tavella, E., Papadopoulos, T.: Expert and novice facilitated modelling: A case of a Viable System Model workshop in a local food network. J. Op. Res. Soc. 66, 247-264 (2014).

9. Recker, J.C., Safrudin, N., Rosemann, M.: How novices design business processes. Information Systems. 37, 557-573 (2012).

10. Powell, S.G., Willemain, T.R.: How novices formulate models. Part I: qualitative insights and implications for teaching. J. Oper. Res. Soc. 983-995 (2007).

11. Franco, L.A.: Rethinking soft OR interventions: models as boundary objects. European Journal of Operational Research. 231, 720-733 (2013).

12. Briggs, R.O., Kolfschoten, G.L., De Vreede, G.J., Lukosch, S., Albrecht, C.C.: Facilitatorin-a-box: process support applications to help practitioners realize the potential of collaboration technology. J. Manage. Inform. Syst. 29, 159-194 (2013).

13. Hjalmarsson, A., Recker, J.C., Rosemann, M., Lind, M.: Understanding the behavior of workshop facilitators in systems analysis and design projects. CAIS. 36, 421-447 (2015).

14. Mayer, R.J.: Models for understanding. (1989).

15. Walls, J.G., Widmeyer, G.R., Sawy, El, O.A.: Building an information system design theory for vigilant EIS. Information systems research. 3, 36-59 (1992).

16. Strauss, A.: Continual Permutations of Action. Aldine de Gruyter, New York (1993).

17. Feldman, M.S., Pentland, B.T.: Reconceptualizing organizational routines as a source of flexibility and change. Administrative Science Quarterly. 48, 94-118 (2003).

18. Herrmann, T., Hoffmann, M., Kunau, G., Loser, K.U.: Modelling Cooperative Work: Chances and Risks of Structuring. Proceedings of COOP 2002. 53-70 (2002).

19. Dix, A., Gongora, L.: Externalisation and design. Presented at the Procedings of the second conference on creativity and innovation in design (2011).

20. Weick, K.E., Sutcliffe, K.M., Obstfeld, D.: Organizing and the process of sensemaking. Organization Science. 16, 409-421 (2005).

21. Frederiks, P.J.M., van der Weide, T.P.: Information modeling: The process and the required competencies of its participants. Data \& Knowledge Engineering. 58, 4-20 (2006).

22. Bider, I., Henkel, M., Kowalski, S., Perjons, E.: Technology Enhanced Learning of Modeling Skills in the Field of Information Systems. Proc. of 8th IADIS IS (2015).

23. Stender, P., Kaiser, G.: Scaffolding in complex modelling situations. ZDM. 47, 1255-1267 (2015).

24. Wood, D., Bruner, J.S., Ross, G.: The role of tutoring in problem solving. Journal of child psychology and psychiatry. 17, 89-100 (1976).

25. Van de Pol, J., Volman, M., Beishuizen, J.: Scaffolding in teacher-student interaction: A decade of research. Educational Psychology Review. 22, 271-296 (2010).

26. Vygotsky, L.S.: Mind in Society. Harvard University Press (1978).

27. Dennen, V.P.: Cognitive apprenticeship in educational practice: Research on scaffolding, modeling, mentoring, and coaching as instructional strategies. Handbook of research on 
educational communications and technology. 2, 813-828 (2004).

28. Jumaat, N.F., Tasir, Z.: Instructional Scaffolding in Online Learning Environment: A Metaanalysis. Proc. of Teaching and Learning in Computing and Engineering (LaTiCE) (2014).

29. Bulu, S.T., Pedersen, S.: Scaffolding middle school students' content knowledge and illstructured problem solving in a problem-based hypermedia learning environment. Educational Technology Research and Development. 58, 507-529 (2010).

30. Su, J.-M.: A self-regulated learning tutor to adaptively scaffold the personalized learning: A study on learning outcome for grade 8 Mathematics. Proc. of UMEDIA 2015 8th (2015).

31. Fretz, E.B., Wu, H.-K., Zhang, B., Davis, E.A., Krajcik, J.S., Soloway, E.: An investigation of software scaffolds supporting modeling practices. Res. Sci. Educ. 32, 567-589 (2002).

32. Sandkuhl, K., Lillehagen, F.: The early phases of enterprise knowledge modelling: practices and experiences from scaffolding and scoping. In: The Practice of Enterprise Modeling. pp. 1-14. Springer (2008).

33. Carstensen, A., Holmberg, L., Sandkuhl, K., Stirna, J.: Integrated Requirements and Solution Modeling: An Approach based on Enterprise Models. Innovations in Information Systems Modeling: Methods and Best Practices. (2008).

34. Oppl, S.: Towards scaffolding collaborative articulation and alignment of mental models. Proceedings of ICKM 2016. (2016).

35. Prusak, N., Hershkowitz, R., Schwarz, B.B.: From visual reasoning to logical necessity through argumentative design. Educational Studies in Mathematics. 79, 19-40 (2012).

36. Ozmantar, M.F., Roper, T.: Mathematical abstraction through scaffolding. Proc. of the 28th Conf. of the International Group for the Psychology of Mathematics Education (2004).

37. Land, S.M., Zembal-Saul, C.: Scaffolding reflection and articulation of scientific explanations in a data-rich, project-based learning environment: An investigation of progress portfolio. Educational Technology Research and Development. 51, 65-84 (2003).

38. Abdu, R., Schwarz, B., Mavrikis, M.: Whole-class scaffolding for learning to solve mathematics problems together in a computer-supported environment. ZDM. 47, 11631178 (2015).

39. Chin, C., Osborne, J.: Students' questions and discursive interaction: Their impact on argumentation during collaborative group discussions in science. Journal of Research in Science Teaching. 47, 883-908 (2010).

40. King, A., Rosenshine, B.: Effects of guided cooperative questioning on children's knowledge construction. The Journal of Experimental Education. 61, 127-148 (1993).

41. Xun, G.E., Land, S.M.: A conceptual framework for scaffolding III-structured problemsolving processes using question prompts and peer interactions. Educational Technology Research and Development. 52, 5-22 (2004).

42. Vogel, F., Wecker, C., Kollar, I., Fischer, F.: Socio-Cognitive Scaffolding with ComputerSupported Collaboration Scripts: a Meta-Analysis. Educ. Psychol. Rev. 1-35 (2016).

43. Kobbe, L., Weinberger, A., Dillenbourg, P., Harrer, A., Hämäläinen, R., Häkkinen, P., Fischer, F.: Specifying computer-supported collaboration scripts. International Journal of Computer-Supported Collaborative Learning. 2, 211-224 (2007).

44. Oppl, S., Alexopoulou, N.: Linking natural modeling to techno-centric modeling for the active involvement of process participants in business process design. IJISMD. 7, (2016). 